\title{
Regional white matter development in very preterm infants: perinatal predictors and early developmental outcomes
}

\author{
Cynthia E. Rogers ${ }^{1,2}$, Tara Smyser ${ }^{1}$, Christopher D. Smyser ${ }^{2,3}$, Joshua Shimony $^{4}$, Terrie E. Inder ${ }^{5}$ and Jeffrey J. Neil ${ }^{6}$
}

BACKGROUND: Preterm infants are at risk for white matter (WM) injury and adverse neurodevelopmental outcomes.

METHODS: Serial diffusion tensor magnetic resonance imaging data were obtained from very preterm infants $(N=78)$ born $<30$ wk gestation imaged up to four times from $26-42 \mathrm{wk}$ postmenstrual age. Slopes were calculated for fractional anisotropy (FA) and mean diffusivity (MD) within regions of interest for infants with $\geq 2$ scans $(N=50)$. Sixty-five children underwent neurodevelopmental testing at $2 \mathrm{y}$ of age.

RESULTS: FA slope for the posterior limb of the internal capsule was greater than other regions. The anterior limb of the internal capsule (ALIC), corpus callosum, and optic radiations demonstrated greater FA slope with increasing gestational age. Infants with patent ductus arteriosus had lower FA slope in the ALIC. MD slope was lower with prolonged ventilation or lack of antenatal steroids. At $2 \mathrm{y}$ of age, lower motor scores were associated with lower FA in the left but higher FA in the right inferior temporal lobe at term-equivalent age. Better social-emotional competence was related to lower FA in the left cingulum bundle.

CONCLUSION: This study demonstrates regional variability in the susceptibility/sensitivity of WM maturation to perinatal factors and relationships between altered diffusion measures and developmental outcomes in preterm neonates.

$P$ reterm infants are at increased risk of brain injury and altered brain development relative to term-born infants, with particular vulnerability in the cerebral white matter (WM). The high rates of WM abnormalities among preterm infants are visible on conventional magnetic resonance imaging (MRI) in the form of diffuse signal abnormality, loss of volume with enlarged ventricles, and delayed maturation (1). The microstructural alterations underlying these WM abnormalities can be assessed using diffusion tensor imaging (DTI), providing quantitative information regarding WM microstructure.

During neonatal brain maturation, fractional anisotropy (FA) increases, while mean diffusivity (MD) decreases in most WM tracts (2). Regional variation in diffusion measures is likely related to varying rates of maturation/myelination, with the corpus callosum and internal capsule maturing earlier than subcortical projection and association pathways (3). Recently, maturation of diffusion parameters in preterm infants with normal neurodevelopment was noted to proceed from central to peripheral and posterior to anterior (4), consistent with previous histology and MRI studies. Preterm infants evaluated at term-equivalent postmenstrual age (PMA) exhibit widespread regional differences in DTI parameters compared to termborn peers (5). These differences have been linked to varied perinatal and clinical factors including chronic lung disease (6), WM injury (7), and postnatal infection (8).

Altered neonatal regional diffusion measures at term-equivalent PMA have been associated with impaired motor (7) and cognitive (7) development at 2 y of age. Trajectory of diffusion measures during the neonatal period, namely, a slower increase in WM FA, has been related to impaired developmental outcomes at 18 mo of age (9). Children born preterm are at risk for poor social-emotional development (10). In prior work, we found evidence that regional diffusion abnormalities at termequivalent age were related to poor social skills at $5 \mathrm{y}$ of age (11).

We expand upon previous work through investigation of WM microstructure maturation during the neonatal intensive care period among a cohort of prematurely born infants scanned longitudinally to (i) identify perinatal clinical factors associated with alterations in the trajectory of WM microstructure maturation and (ii) relate regional WM microstructure at termequivalent age to developmental deficits among preterm infants at $2 \mathrm{y}$ of age, including social-emotional outcomes. We hypothesized that the trajectory of FA and MD would vary regionally with early myelinating regions having steeper increases in FA and decreases in $\mathrm{MD}$; detrimental clinical factors would slow the anticipated increase in FA and decrease in MD; and altered regional diffusion parameters at term-equivalent age would be related to neurodevelopmental outcomes at $2 \mathrm{y}$ of age.

\section{RESULTS}

Eighty-two very-preterm (VPT) infants underwent DTI at termequivalent PMA, with 80 infants surviving. Characteristics of

\footnotetext{
'Department of Psychiatry, Washington University School of Medicine, St Louis, Missouri; ${ }^{2}$ Department of Pediatrics, Washington University School of Medicine, St Louis, Missouri; ${ }^{3}$ Department of Neurology, Washington University School of Medicine, St Louis, Missouri; ${ }^{4}$ Mallinckrodt Institute of Radiology, Washington University School of Medicine, St Louis, Missouri; ${ }^{5}$ Department of Pediatric Newborn Medicine, Brigham and Women's Hospital, Boston, Massachusetts; ${ }^{6}$ Department of Neurology, Boston Children's Hospital, Boston, Massachusetts. Correspondence: Cynthia E. Rogers (rogersc@psychiatry.wustl.edu)

Received 17 January 2015; accepted 29 June 2015; advance online publication 14 October 2015. doi:10.1038/pr.2015.172
} 
the remaining cohort are displayed in Table 1 . Those with only a term-equivalent scan $(N=19)$ or with only two scans less than $3 \mathrm{wk}$ apart $(N=9)$ were excluded from the serial analyses. Among the remaining data, scans from an additional two subjects were removed because of poor data quality, leaving 50 subjects. There were no significant differences between those included or excluded with respect to sex, ethnicity, and presence of WM injury, perinatal factors, or developmental measures evaluated at $2 \mathrm{y}$ of age.

\section{Perinatal Factors and Serial Diffusion Values}

Mixed-models results of the impact of perinatal clinical factors on serial regional diffusion measures are summarized in Table 2. Nonsignificant factors were removed from the model, including necrotizing enterocolitis, hemisphere, sex, and ethnicity. There was a main effect of region, with post hoc comparisons finding slope of FA in the posterior limb of the internal capsule (PLIC) was greater than that in other regions after adjusting for perinatal factors $(P<0.001)$ (Figure 1$)$. To examine the relationship between the rate of WM maturation and $\mathrm{FA}$ at term-equivalent age, regional slope and the associated term-equivalent FA value were correlated via partial correlations, adjusting for PMA at term-equivalent scan. The PLIC had the weakest correlation of any region $(r=0.47, P<0.001$; range of all other regions of interest (ROIs) $r=0.50-0.76$, $P<0.001$ ), though all correlations were significant after correction for multiple comparisons. There was a main effect of sepsis $(P=0.01)$ and antenatal steroids $(P=0.04)$ on FA slope. Lower FA slopes were found in infants with sepsis (no sepsis: slope $=0.0087$ vs. sepsis: slope $=0.0052)$ and infants whose mothers received antenatal steroids (no antenatal steroids: slope $=0.0084$ vs. antenatal steroids: slope $=0.0055$ ). These relationships with sepsis and antenatal steroids remained when grouping ROIs by myelination rate (see Supplementary Information online).

ROI by birth gestational age (GA) mixed model interaction term was significant for FA $(P=0.01)$, reflecting regional differences in the impact of GA at birth. The anterior limb of the internal capsule (ALIC) $(P=0.01)$, corpus callosum $(P=$ $0.004)$, and optic radiations $(P=0.004)$ were the regions with values related to GA, demonstrating an increasing slope with increasing GA at birth. There was an ROI by patent ductus arteriosus (PDA) treatment interaction in the ALIC $(P=0.02)$, with those medically treated for PDA having a lower FA slope than those without a PDA ( 0.0030 vs. 0.0087 , respectively).

For MD, there was not a main effect of ROI or hemisphere. There was an effect of maternal receipt of antenatal steroids $(P=0.002)$ and of length of endotracheal ventilation $(P$ $=0.008)$ on the overall slope. $\mathrm{MD}$ declined more rapidly in infants who did not receive antenatal steroids (no antenatal steroids: slope $=-0.0269$ vs. antenatal steroids: slope $=-0.0064$ ) or had prolonged ventilation (longer ventilation: slope = -0.0246 vs. shorter ventilation: slope $=-0.0088)$. Region did not have a significant interaction with antenatal steroids $(P=$ $0.12)$ or prolonged ventilation $(P=0.54)$. On initial MRI scan, children whose mothers did not receive antenatal steroids had
Table 1. Sample characteristics

\begin{tabular}{|c|c|}
\hline Characteristic & $N=78$ \\
\hline Birth weight, g, mean (SD) & $941(246)$ \\
\hline GA, weeks, mean (SD) & $26.6(1.8)$ \\
\hline \multicolumn{2}{|l|}{ Number of MRI scans, $n(\%)$} \\
\hline One scan & $19(24)$ \\
\hline Two scans & $29(37)$ \\
\hline Three scans & $23(30)$ \\
\hline Four scans & $7(9)$ \\
\hline GA at first serial MRI, weeks, mean (SD) $N=50$ & $30.4(2.4)$ \\
\hline GA at term-equivalent MRI, weeks, mean (SD) & $37.6(1.5)$ \\
\hline Male, $n(\%)$ & $33(42)$ \\
\hline \multicolumn{2}{|l|}{ Ethnicity, $n(\%)$} \\
\hline African American & $33(42)$ \\
\hline Caucasian & $41(53)$ \\
\hline Asian & $3(4)$ \\
\hline Hispanic & $1(1)$ \\
\hline No antenatal steroid, $n(\%)$ & $12(15)$ \\
\hline Days of ventilation, mean, median, (IQR) & $12.8,2(1-14)$ \\
\hline Days on TPN, mean, median, (IQR) & $25.6,17(11-32)$ \\
\hline PDA treated with NSAID, $n(\%)$ & $30(39)$ \\
\hline IUGR, $n(\%)$ & $5(6)$ \\
\hline Dexamethasone, $n(\%)$ & $8(10)$ \\
\hline Change in SDS at birth, $M(S D)$ & $-0.89(0.8)$ \\
\hline $\mathrm{NEC}, n(\%)$ & $6(8)$ \\
\hline Sepsis, $n(\%)$ & $23(30)$ \\
\hline Term-equivalent white matter injury, $n(\%)^{\text {a }}$ & $16(21)$ \\
\hline Age 2 outcomes & $N=65$ \\
\hline Bayley III cognitive, mean (SD) & $86.8(9.4)$ \\
\hline Normal, $n(\%)$ & $20(31)$ \\
\hline Mild, $n(\%)$ & $31(48)$ \\
\hline Moderate, $n(\%)$ & $13(20)$ \\
\hline Severe, $n(\%)$ & $1(2)$ \\
\hline Bayley III language, mean (SD) & $89.9(11.1)$ \\
\hline Normal, $n(\%)$ & $16(25)$ \\
\hline Mild, $n(\%)$ & $37(57)$ \\
\hline Moderate, $n(\%)$ & $11(17)$ \\
\hline Severe, $n(\%)$ & $1(2)$ \\
\hline Bayley III motor, mean (SD) & $84.6(10.6)$ \\
\hline Normal, $n(\%)$ & $9(14)$ \\
\hline Mild, $n(\%)$ & $34(52)$ \\
\hline Moderate, $n(\%)$ & $17(26)$ \\
\hline Severe, $n(\%)$ & $5(8)$ \\
\hline ITSEA competence domain, mean (SD) & $42.7(12.8)$ \\
\hline Of concern, $n(\%)$ & $18(28)$ \\
\hline
\end{tabular}

Children were classified based on their Bayley-lll composite scores into the following categories: normal ( $\geq 95)$, mild ( $<95$ and $\geq 80$ ), moderate ( $<80$ and $\geq 65$ ), and severe impairment (<65). ITSEA of concern means T scored in the lowest 10th percentile (most impaired).

IQR, interquartile range; IUGR, intrauterine growth restriction; NEC, necrotizing enterocolitis; NSAID, nonsteroidal anti-inflammatory drug (indomethacin or ibuprofen): SDS, standardized weight for length; TPN, total parenteral nutrition; WM, white matter. ancludes focal and cystic WM lesions as well as Grades III and IV intraventricular hemorrhage. 
Table 2. Results from mixed-effects regression model examining impact of clinical factors on regional FA and MD development among preterm infants

\begin{tabular}{|c|c|c|c|c|c|c|}
\hline \multirow[b]{2}{*}{ Factor } & \multicolumn{3}{|c|}{ FA } & \multicolumn{3}{|c|}{ MD } \\
\hline & DF & $F$ & $P$ value & DF & $F$ & $P$ value \\
\hline Hemisphere & 1 & 0.06 & 0.81 & 1 & 2.48 & 0.12 \\
\hline $\mathrm{ROI}$ & 9 & 2.60 & 0.005 & 9 & 1.18 & 0.30 \\
\hline GA & 1 & 0.01 & 0.93 & 1 & 1.42 & 0.32 \\
\hline Antenatal steroids & 1 & 4.23 & 0.04 & 1 & 9.78 & 0.002 \\
\hline SDS change & 1 & 0.18 & 0.67 & 1 & 0.09 & 0.77 \\
\hline Dexamethasone & 1 & 0.93 & 0.34 & 1 & 0.00 & 0.99 \\
\hline $\begin{array}{l}\text { Length of } \\
\text { ventilation }\end{array}$ & 1 & 0.42 & 0.52 & 1 & 6.73 & 0.01 \\
\hline Length of TPN & 1 & 0.76 & 0.38 & 1 & 0.36 & 0.55 \\
\hline PDA Med Tx & 1 & 1.16 & 0.28 & 1 & 0.00 & 0.97 \\
\hline Sepsis & 1 & 6.57 & 0.01 & 1 & 0.65 & 0.42 \\
\hline White matter injury & 1 & 0.82 & 0.36 & 1 & 0.36 & 0.55 \\
\hline ROI $\times$ hemisphere & 8 & 0.65 & 0.74 & 8 & 0.84 & 0.57 \\
\hline $\mathrm{ROI} \times \mathrm{GA}$ & 9 & 2.42 & 0.01 & 9 & 1.17 & 0.31 \\
\hline $\begin{array}{l}\mathrm{ROI} \times \text { antenatal } \\
\text { steroids }\end{array}$ & 9 & 1.58 & 0.11 & 9 & 1.67 & 0.09 \\
\hline $\mathrm{ROI} \times \mathrm{SDS}$ change & 9 & 0.88 & 0.53 & 9 & 1.54 & 0.13 \\
\hline $\begin{array}{l}\mathrm{ROI} \times \\
\text { dexamethasone }\end{array}$ & 9 & 0.68 & 0.72 & 9 & 1.69 & 0.09 \\
\hline $\begin{array}{l}\text { ROI } \times \text { length of } \\
\text { ventilation }\end{array}$ & 9 & 1.33 & 0.26 & 9 & 0.84 & 0.58 \\
\hline ROI $\times$ length of TPN & 9 & 0.62 & 0.44 & 9 & 0.74 & 0.91 \\
\hline ROI $\times$ PDA Med Tx & 9 & 2.13 & 0.03 & 9 & 0.79 & 0.63 \\
\hline $\mathrm{ROI} \times$ sepsis & 9 & 0.85 & 0.57 & 9 & 1.88 & 0.05 \\
\hline $\begin{array}{l}\text { ROI } \times \text { white matter } \\
\text { injury }\end{array}$ & 9 & 0.56 & 0.84 & 9 & 0.87 & 0.56 \\
\hline
\end{tabular}

$\mathrm{FA}$, fractional anisotropy; $\mathrm{GA}$, gestational age; $\mathrm{MD}$, mean diffusivity; $\mathrm{PDA}$, patent ductus arteriosus; ROI, region of interest; SDS, standardized weight for length; TPN, total parenteral nutrition; $\mathrm{Tx}$, treatment.

greater global mean MD after adjusting for PMA at scan compared to infants whose mothers received steroids (adjusted mean: 1.71, SD: 0.09 and 1.61, SD 0.06, respectively, $P=0.001$ ), with no impact of prolonged ventilation (adjusted mean 1.64, SD: 0.07 and 1.62, SD: 0.07, respectively, $P=0.41$ ). By termequivalent age, there was no difference in MD between infants with or without or prolonged ventilation (adjusted mean: 1.56, SD: 0.05 and 1.58, SD: 0.05 , respectively, $P=0.24$ ) or who did or did not receive antenatal steroids (adjusted mean: 1.58, SD: 0.05 and 1.55 , SD: 0.05 , respectively, $P=0.18$ ). These associations were also significant when the analysis was repeated with ROIs grouped by myelination rate (see Supplementary Information online).

\section{Association Between Neonatal Diffusion Measures and Age 2 Developmental Outcomes}

Sixty-five infants underwent developmental assessments at 2 y of age. After conducting stepwise regression analyses using $\mathrm{FA}$ and $\mathrm{MD}$, the following diffusion measures were candidate variables for the neurodevelopmental assessments on the Bayley-III: cognitive composite (MD left ALIC), language composite (MD left ALIC), motor composite (FA left and right inferior temporal lobe, FA right ALIC, MD left ALIC), and Infant Toddler Social Emotional Assessment (ITSEA) competence domain (FA left cingulum and MD left PLIC).

Table 3 displays results of the linear mixed models relating neurodevelopmental measures at $2 \mathrm{y}$ of age to regional neonatal diffusion measures at term-equivalent age. Models were adjusted for term-equivalent PMA and sex. Lower MD in the left ALIC was related to impaired performance on the BayleyIII language composite scale $(P=0.04, \alpha=0.05 / 1=0.05)$, however, FA in the left ALIC was not $(P=0.91)$. Higher FA in the left inferior temporal lobe was related to higher motor composite scores $(P=0.004, \alpha=0.05 / 4=0.013)$. Conversely, lower FA in the right inferior temporal lobe was associated with higher motor composite scores $(P=0.004)$. MD in neither the left $(P=0.17)$ nor right temporal lobe $(P=0.97)$ was related to motor composite scores. Post hoc paired $t$-test analysis of FA in the left and right inferior temporal lobe demonstrated hemispheric asymmetry, with higher values on the left than right (mean: 0.133 and $0.126, P=0.04$ ). There was a correlation between hemispheric asymmetry and motor composite scores $(r=0.43, P<0.001)$. This relationship was stronger for the fine motor subscale $(r=0.46, P<0.001)$ than the gross motor subscale $(r=0.29, P=0.02)$. Birth GA was not a mediator of this relationship $(P=0.68)$ and did not alter the relationship between inferior temporal lobe FA and motor outcome. When children were grouped based on motor performance into normal, mild, moderate, or severe impairment, lesser impairment was associated with greater hemispheric asymmetry in FA between the left and right inferior temporal lobe $(F=3.75$, $P=0.015$; Figure 2). Further, for those with both serial scans and outcome data $(N=38)$, the difference in the slope of FA between the left and right inferior temporal lobe was related to the motor composite score after adjusting for sex and PMA at scan $(P=0.04)$, with those with FA increasing more rapidly on the left relative to the right inferior temporal lobe having higher motor scores.

Post hoc analyses examined the radial and axial diffusivities in the inferior temporal lobe and related them to the BayleyIII motor composite score. After adjusting for PMA at scan and sibling correlation, both higher axial $(P=0.04)$ and lower radial diffusivity $(P=0.02)$ in the left temporal lobe and lower axial $(P=0.04)$ and higher radial diffusivity $(P=0.02)$ in the right temporal lobe were associated with Bayley-III motor composite scores.

Lower ITSEA competence scores were related to higher FA in the left cingulum $(P=0.001 ; \alpha=0.05 / 2=0.025$; Figure 3$)$ but were not related to MD in the left cingulum $(P=0.08)$. We also evaluated the role of $\mathrm{AD}$ and $\mathrm{RD}$ when examining termequivalent FA in the cingulum and ITSEA competence scores. Lower $\mathrm{RD}(P=0.002)$, but not higher $\mathrm{AD}(P=0.12)$, was associated with lower ITSEA competence scores after adjustment for PMA at scan and sibling correlation. For infants with both serial scans and ITSEA competence scores $(N=38)$, there was 


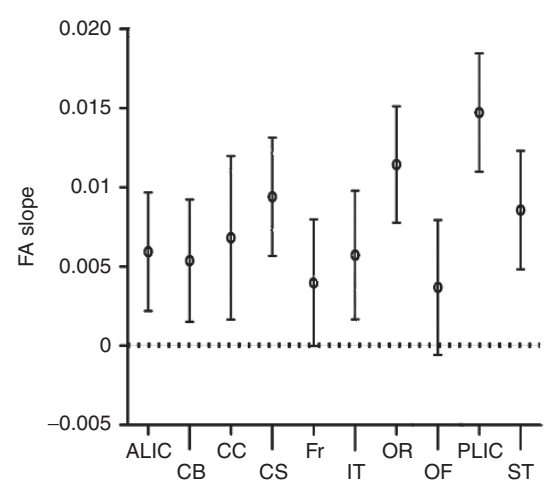

d

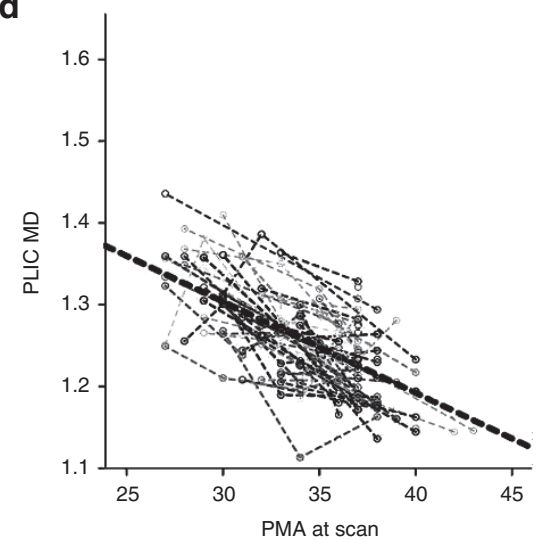

b

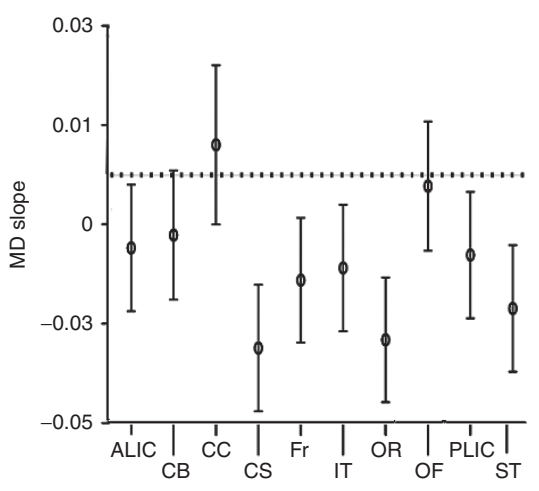

e

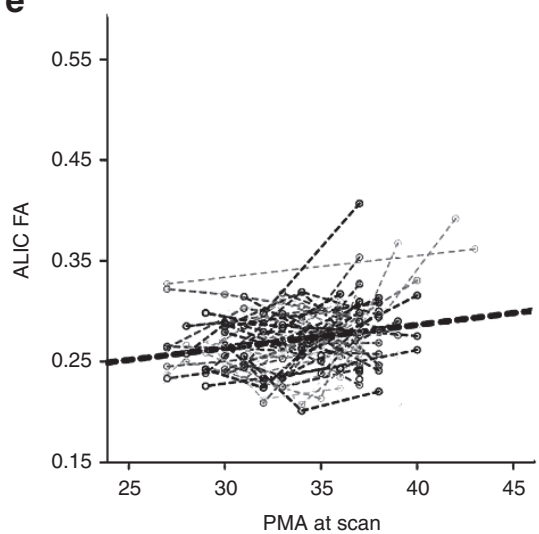

C

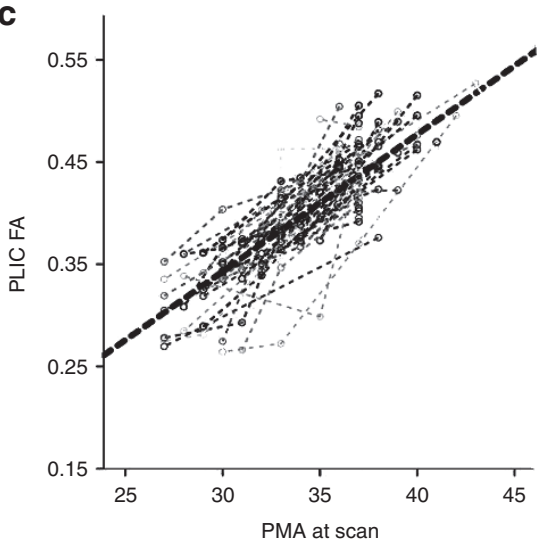

f

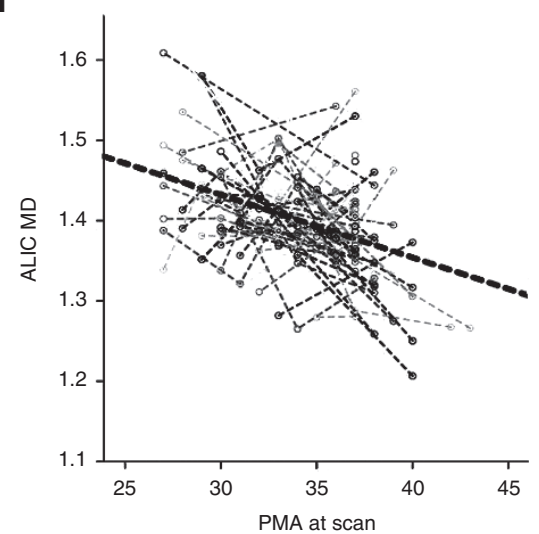

Figure 1. Plots of the slope of (a) fractional anisotropy (FA) and (b) mean diffusivity (MD) for each region of interest. The dotted line at zero delineates positive or negative slopes. ALIC, anterior limb of the internal capsule; $\mathrm{CB}$, cingulum bundle; CC, corpus callosum; $\mathrm{CS}$, centrum semiovale; $\mathrm{Fr}$, frontal lobe; IT, inferior temporal lobe; OR, optic radiations; OF, orbitofrontal; PLIC, posterior limb of the internal capsule; ST, superior temporal lobe. Middle panels: trajectory of (c) FA and (d) MD of the PLIC for each infant, with group mean denoted by the dotted black line. Lower panels: trajectory of (e) FA and (f) MD for the ALIC.

a significant correlation between the trajectory of FA in the left cingulum and ITSEA competence scores $(r=-0.36, P=0.03)$.

\section{DISCUSSION}

We analyzed the regional WM microstructural development of a cohort of VPT infants and related regional diffusion maturation to perinatal clinical factors, confirming that GA, antenatal steroid exposure, sepsis, length of ventilation, and PDA were correlated with a change in diffusion measures during neonatal intensive care unit hospitalization, often in a regionally specific manner. We also demonstrated that regional diffusion measures at term-equivalent age were associated with specific early developmental outcomes at $2 \mathrm{y}$ of age.

Many findings from this study support the previously reported pattern of changes in diffusion measures during WM development, with FA increasing and MD decreasing as PMA increases (with some exceptions, vide infra). These changes in FA and MD are likely driven by a number of factors, most notably myelination (12). Regional variations in FA and MD reflect varying rates of myelination (13), though myelination rates may also be affected by injury, particularly to cells from the oligodendrocyte lineage. This injury would be expected to slow the increase in FA and decrease in MD associated with maturation. An important caveat is that in many areas where myelination has not occurred by term-equivalent age, anisotropy values are low, sometimes at the noise floor. For our data, the noise floor was $\sim 0.02$. For areas that have values at or near this noise floor, disruption of myelination cannot drive the measured value lower thus, anisotropy values are relatively "insensitive" to injury. For areas which are already myelinating (e.g., PLIC), disruption of myelination can lead to lower anisotropy values, rendering them sensitive to injury.

Other neurodevelopmental processes in addition to myelination are relevant. The ALIC is not myelinated at term-equivalent PMA yet has relatively high anisotropy values. These higher anisotropy values result from close packing of axons passing through the ALIC as they connect cortical regions and the thalamus. Reduction in FA most likely reflects injury to axons and their packing rather than myelin disruption. As the ALIC carries axons from a variety of cortical areas that communicate with the thalamus, it may serve to integrate injury to these areas.

Our finding that the slope of PLIC FA values was steeper than other regions likely reflects the early myelination of the PLIC (14). While regions with greater slopes tended to have higher FA values at term-equivalent PMA, this was not the 
Table 3. Association between neonatal term-equivalent diffusion measures and age 2 developmental outcomes

\begin{tabular}{lc}
\hline Developmental test & Adjusted standardized estimate $(95 \% \mathrm{Cl})$ \\
\hline Bayley III cognitive composite & $-0.2(-2.3$ to 1.8$)$ \\
MD left ALIC & \\
Bayley III language composite & $3.0(0.2 \text { to } 5.9)^{*}$ \\
MD left ALIC & \\
Bayley III motor composite & $-3.9(-6.4 \text { to }-1.3)^{* *}$ \\
FA right inferior temporal & $3.2(0.7 \text { to } 5.7)^{*}$ \\
FA left inferior temporal & $-2.4(-4.9$ to 0.1$)$ \\
FA right ALIC & $0.2(-2.2$ to 2.6$)$ \\
MD left ALIC & \\
ITSEA competence & $-4.4(-7.5 \text { to }-1.3)^{* *}$ \\
FA left cingulum & $2.6(-0.5$ to 5.7$)$ \\
MD left PLIC &
\end{tabular}

ALIC, anterior limb of the internal capsule; $\mathrm{Cl}$, confidence interval; $\mathrm{FA}$, fractional anisotropy; ITSEA, Infant Toddler Social Emotional Assessment; MD, mean diffusivity: PLIC, posterior limb of the internal capsule. ${ }^{*} P<0.05 ;{ }^{* *} P<0.01$

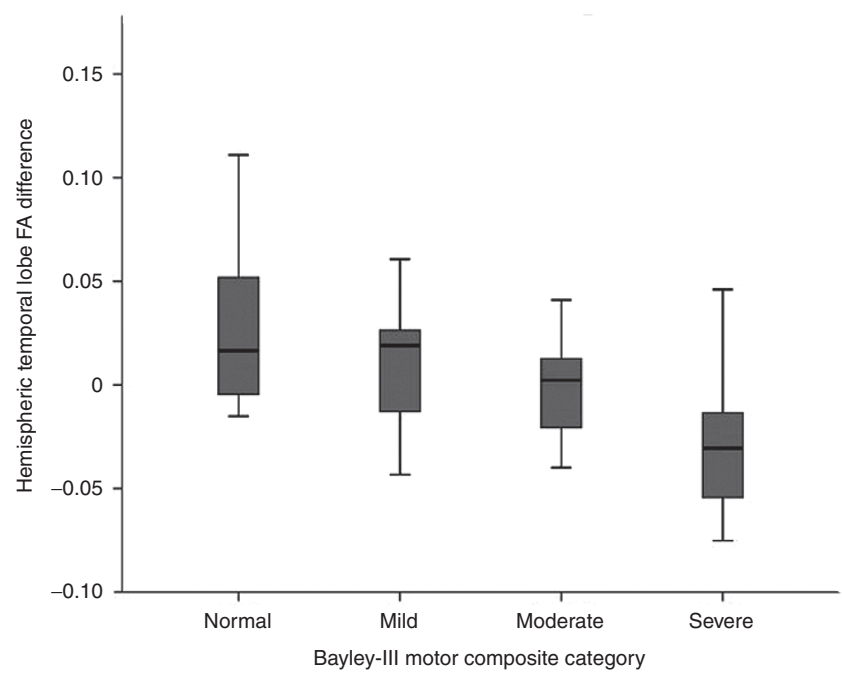

Figure 2. Boxplots of hemispheric asymmetry between left and right inferior temporal fractional anisotropy (FA) and Bayley-III Motor composite categories.

case within the PLIC. The PLIC's FA values at term-equivalent PMA tended to reflect ranking relative to other infants, with similar slopes between infants (Figure 3c). This suggests similar rates of development in the PLIC among all infants.

The relationship between earlier GA at birth and lower slope of FA for areas with relatively high FA values, the corpus callosum, optic radiations, and ALIC, was consistent with prior work. Previous investigations linked lower GA among preterm infants to altered diffusion measures at term-equivalent PMA (7), including in the optic radiations (15), though this was not noted in the corpus callosum (16). Others have noted no impact of extreme prematurity on diffusion maturation when compared to preterm infants born at later ages when controlling for other clinical variables (17). Our study differed as we evaluated additional regions, including two of the three areas where degree of prematurity was influential in our study, the corpus callosum, and the ALIC. We also analyzed GA as a continuous variable and had many infants with more than two scans. Nevertheless, we identified an effect of GA in only a limited number of regions. While acknowledging the technical considerations discussed earlier, this may reflect regionspecific vulnerability to injury. However, the regions where we find injury do not necessarily correspond to the periventricular WM shown in histologic studies to be vulnerable (18).

We also found that sepsis and PDA affected the trajectory of FA. Sepsis did so independent of region. Our findings here and from other cohorts (8) indicate that sepsis potentially affects WM development through the deleterious impact of related inflammatory processes on the premyelinating oligodendrocytes (19), disrupting their development and ensheathment of axons (20). Similarly, PDA resulted in a threefold lower slope of FA in the ALIC. Earlier work has linked PDAs with WM injury (1), indicating WM development may be vulnerable to the effects of PDA. Similarly, a hemodynamically significant PDA can decrease cerebral oxygenation and tissue oxygen extraction, potentially resulting in neurologic injury (21). It is not clear why the ALIC is particularly susceptible to the influence of PDA, though, as noted above, it is unique among the areas studied. In contrast, a longer duration of indomethacin for PDA therapy was associated with less WM injury (22).

Some findings did not support the theory that a steeper rise in FA and decline in MD are indicative of healthy WM maturation. Lack of maternal receipt of antenatal steroids and prolonged mechanical ventilation were associated with a more rapidly declining slope in MD and lack of antenatal steroids was also modestly related to a more rapidly accelerating slope in FA (effects that persisted even after we collapsed the ROIs into three groups based on the relative order of myelination). Prenatal steroids are administered to accelerate lung maturation, and their administration may also affect brain maturation, at least in terms of water content, which declines with brain maturation. Infants not exposed to antenatal steroids had higher initial MD values, consistent with higher brain water content (less mature brain) compared with those who were exposed explaining the difference in slopes, as MD values were similar at term-equivalent PMA. It is unclear how prolonged mechanical ventilation would lead to more rapid declines in MD values, though the differences between those with and without prolonged ventilation were modest.

Similarly, impairment in two developmental measures at $2 \mathrm{y}$ of age was associated with higher FA in two WM regions. Impaired social-emotional competence on the ITSEA was associated with higher FA in the left cingulum, primarily related to lower radial diffusivity. This domain assesses several core symptoms of autism spectrum disorder (ASD), including empathy, imitative play, prosocial behavior, and attention. ITSEA subscales are markedly abnormal in children with ASD (23), and the cingulum bundles are one of the most abnormal WM regions in those with autism (24). They are one of the 
a

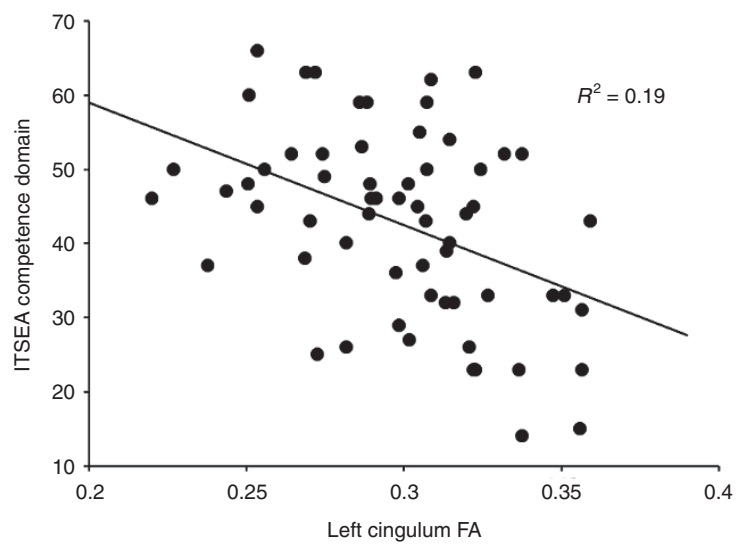

b

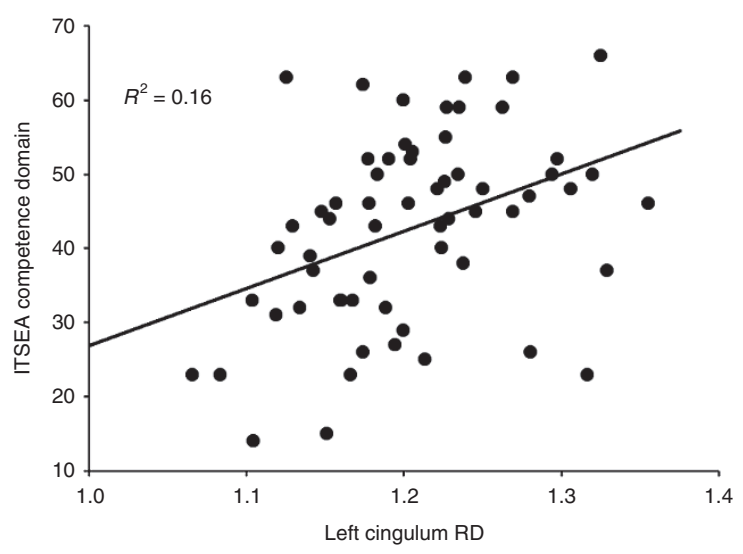

c

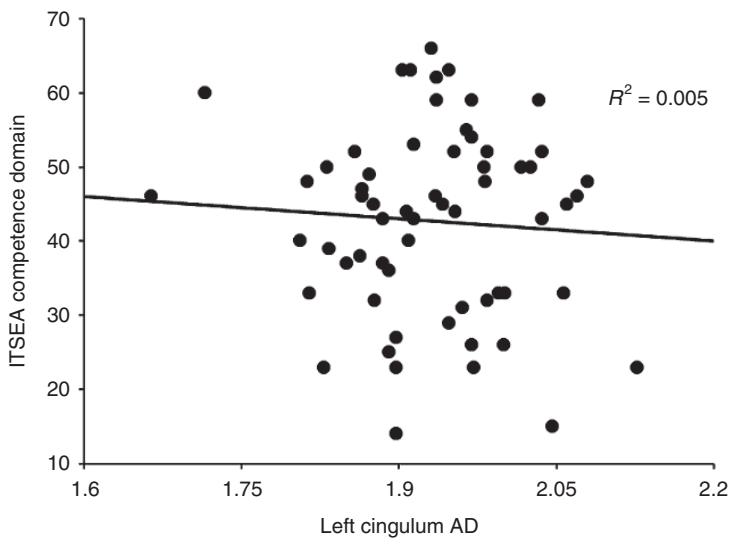

Figure 3. (a) Regression plot demonstrating the relationship between fractional anisotropy (FA) in the left cingulum at term-equivalent age and ITSEA competence scores at $2 \mathrm{y}$ of age. (a) Note the association between more impaired (lower) ITSEA competence scores and higher FA $(P=0.001)$. This relationship appears to be related to $(\mathbf{b})$ lower radial diffusivity $(P=0.001)$ and $(\mathbf{c})$ not higher axial diffusivity $(P=0.15)$. ITSEA, Infant Toddler Social Emotional Assessment.

major WM tracts connecting intrahemispheric association regions like the cingulate cortex with limbic structures in the temporal lobe (24), and abnormalities of the temporal lobe have been associated with both the social impairment (25) and executive function deficits of those with ASD (26). Prior work investigating other WM regions demonstrated higher FA associated with ASD symptoms. Higher FA was reported in the WM of 6-mo-old infants who later developed ASD (27) and

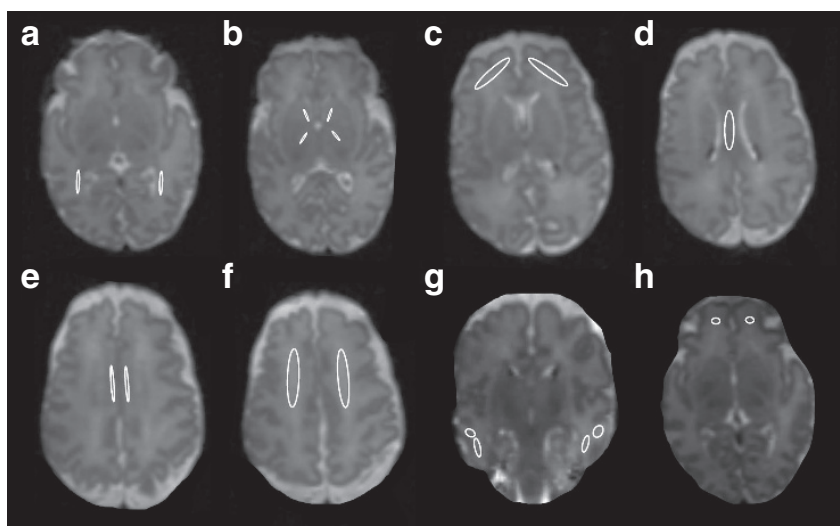

Figure 4. Regions of interest overlayed on term-equivalent $\mathrm{T} 2$-weighted images located in the (a) optic radiations, (b) anterior and posterior limbs of the internal capsules, (c) frontal lobes (forceps minor), (d) corpus callosum, (e) cingulum bundles, (f) centrum semiovale, (g) superior temporal lobes (anterior) and inferior temporal lobes (posterior) and (h) orbitofrontal region.

specifically in the cingulum of young children with ASD (28). Our finding was specific to the left cingulum bundle, which is consistent with prior work reporting higher FA predominantly in regions in the left hemisphere among toddlers with ASD (29), though other studies have reported higher FA bilaterally $(27,28)$. Higher anisotropy in WM regions in school-age preterm children was related to more impaired social-emotional development (30). Our data suggest that these changes are already present early in infancy. Nevertheless, research that extends to older ages with more definitive assessments of ASD is required to support our preliminary findings and is currently underway with our cohort.

Likewise, higher FA in the right inferior temporal lobe was related to impairment in motor scores, particularly fine motor scores. This higher anisotropy can be attributed to both an increase in axial and decrease in radial diffusivities. This result may paradoxically reflect the pattern typically characteristic of impaired maturation, as higher FA in the right inferior temporal lobe was paired with concomitantly lower FA in the left. Children with the opposite pattern of asymmetry, higher FA on the left and lower on the right, had better motor scores. This pattern of hemispheric asymmetry in diffusion parameters, higher FA on the left and lower on the right, is likely normal, as it has been previously reported in healthy subjects in temporal lobe WM (31), even as early as infancy (32). Further, loss of this asymmetry in FA has been noted in preterm children with developmental deficits (33).

While the temporal lobe is not typically associated with fine motor skills, the inferior temporal lobe ROI used in this study included fibers from the inferior longitudinal fasciculus (ILF). In very-low-birth-weight adolescents, impairment in fine motor scores has been linked with lower FA in the left ILF (34), similar to the current study. Further, diffusion parameters in the ILF have been linked to impairments in visuomotor speed and dexterity (35), both of which are part of the Bayley-III fine motor scale, which assesses visuospatial abilities and perceptual motor integration. Disruption of the microstructural 
integrity of the ILF may cause impairments in these functions as it serves as a pathway connecting the anterior temporal lobe and visual cortex (36) and has been linked to the cerebral visual impairment noted in VPT infants (37). This impairment can include a deficit in visual guidance of upper limbs, thereby affecting fine motor skills.

Though it is unclear why FA values thought to typically represent more robust WM maturation were linked with detrimental perinatal factors and impaired social-emotional development, several theories have been offered. Higher FA may reflect greater myelination and/or a greater number of WM fibers. It may also indicate reduced branching, smaller axonal diameter, or fewer fibers crossing the tract of interest, findings more consistent with aberrant development (38). The higher FA in the cingulum linked to poor outcome was accompanied by lower RD which is consistent with aforementioned explanations. Indeed, higher FA and/or lower MD in WM has been noted in pathological conditions including autism (27) and preterm infants (39). In a study showing higher regional FA values in WM of preterm school-age children, it was postulated that the higher FA could represent a compensatory change to offset regions with weaker structural connections (30). It is unclear whether such compensation could occur as early as the neonatal period.

While there are many strengths of the current study, including its prospective design, large proportion of infants with three or more MRI scans, and comprehensive assessment of perinatal clinical factors and early child development, there are potential limitations as well. The overall size of the cohort with serial scans, while comparable in size to some recent work (4), was smaller, potentially explaining some differences in the findings (17). The reliance solely on parental report for assessment of social-emotional competence is a limitation, though parental reports of social-emotional problems during early childhood do predict future childhood psychiatric diagnoses in preterm children (10). Another potential limitation is the use of ROI analysis, which has risk of subjectivity and poor reproducibility, though our inter-rater reliability for ROIs was high (mean: 0.88). ROI analysis has the advantage of providing greater power to detect differences, as only regions chosen a priori are tested in the models. The specificity provided through manual placement of ROIs allows the examination of regions that might be inaccessible through methods like MR tractography or Tract-Based Spatial Statistics, as the algorithms used for creating tracts choose, of necessity, areas of high anisotropy, potentially ignoring WM areas with FA values below a set threshold. This effect would be more notable in a premature population scanned at very early PMA for whom FA values are low.

A relative strength of the DTI data in the current study is the higher spatial resolution (voxel size $1.2 \times 1.2 \times 1.2 \mathrm{~mm}^{3}$ ) and greater dimensionality (31-48 b vectors) than many prior studies. High spatial resolution is imperative for limiting partial volume averaging effects. Additionally, higher dimensionality improves reliability of diffusion parameter estimates. Also, placing ROIs on individual native space images allows for directed examination and individualization of ROI placement.
This method likely improves the accuracy of measured values and sensitivity to altered microstructure.

In summary, this study highlights regional alterations in WM microstructural development in VPT infants with differential susceptibility to specific perinatal factors during the neonatal intensive care unit hospitalization. Understanding how perinatal exposures affect the maturation of specific WM regions may enable targeted surveillance of infants. Further, we found that regional DTI measures were associated with early developmental outcomes. Thus, DTI may be a useful marker for developmental delays frequently found in preterm children, allowing early identification of regional WM abnormalities associated with developmental impairment. The findings highlight the subtlety required to interpret DTI values in the face of potential injury and the varying regional sensitivity of DTI to disruption of development. While requiring both replication and extension to later childhood with longitudinal follow-up, neonatal DTI may provide valuable prognostic information and assist in targeted surveillance and intervention in vulnerable preterm children.

\section{METHODS}

\section{Participants}

VPT infants were recruited from a level III neonatal intensive care unit in St Louis, Missouri, between 2007 and 2010. Infants born less than $30 \mathrm{wk}$ gestation were eligible except those with chromosomal abnormalities or proven congenital infections (e.g., cytomegalovirus, toxoplasma, rubella). Of 174 eligible infants, 154 were approached and 136 were recruited. Parental informed consent was obtained for each subject prior to participation in the study. Eight withdrew and one was excluded after a congenital anomaly was diagnosed following enrollment. Twenty infants died during the neonatal intensive care unit course. Two additional infants died prior to follow-up testing. The study was approved by the Washington University Human Research Protection Office.

\section{Imaging Protocol}

Infants were imaged without sedating medications while asleep or resting quietly up to four times from 26-42 wk PMA at designated intervals $(<30,30-31,34-35$, and $36-40 \mathrm{wk}$ PMA) if they were clinically stable to travel to the MRI suite. Images were acquired on a 3T Tim Trio system (Siemens, Erlangen, Germany). Structural images included a T1-weighted sagittal, magnetization-prepared rapid gradient echo sequence (repetition time $(\mathrm{TR})=1550 \mathrm{~ms}$, inversion time $(\mathrm{TI})=1,100 \mathrm{~ms}$, echo time $(\mathrm{TE})=3.05 \mathrm{~ms}$, flip angle $=15^{\circ}$, $1 \times 1 \times 1.25 \mathrm{~mm}^{3}$ voxels) and a T2-weighted fast spin echo sequence $\left(\mathrm{TR}=8,210 \mathrm{~ms}\right.$, TE $=161 \mathrm{~ms}, 1 \times 1 \times 1 \mathrm{~mm}^{3}$ voxels $)$. The diffusionweighted sequence acquisition parameters included $128-\mathrm{mm}$ FoV, $\mathrm{TR}=13,300 \mathrm{~ms}, \mathrm{TE}=112 \mathrm{~ms}, 1,266 \mathrm{~Hz} / \mathrm{Px}$ bandwidth, voxel size $1.2 \times 1.2 \times 1.2 \mathrm{~mm}^{3}$ and $31-48 \mathrm{~b}$ amplitudes and directions ranging from 0 to $1,200 \mathrm{~s} / \mathrm{mm}^{2}$. Total acquisition time for all sequences was $\sim 60 \mathrm{~min}$.

\section{Diffusion Processing}

The diffusion signal attenuation curve was modeled as a monoexponential function plus a constant, and diffusion parameters were estimated using Bayesian probability theory (40). Maps of FA, MD, radial diffusivity, and axial diffusivity were generated, as were red-green-blue color maps. Bilateral ROIs were placed manually on native diffusion parametric maps by three investigators using FA, MD, and red-greenblue maps. Mean inter-rater reliability for all regions was 0.88 for MD and 0.87 for FA (range: $0.70-0.99$ ). The ROIs were evaluated on three contiguous slices in order to minimize through-slice partial volume averaging. A priori rROIs were placed in the: ALIC, PLIC, optic radiations, corpus callosum, cingulum bundle, centrum semiovale, frontal lobe in the forceps minor and subcortical WM of the superior temporal 
lobe, inferior temporal lobe, and orbitofrontal region (Figure 4). ROIs were sampled using ANALYZE 10.0 software (Rochester, MN) in combination with laboratory-written analysis scripts.

\section{Clinical Factors}

Infant medical course information was prospectively obtained from the infant and maternal medical record, including: gestational age (GA) at birth, culture-positive sepsis (dichotomized yes/no), maternal antenatal steroids (dichotomized yes/no), postnatal steroid use (dichotomized yes/no), length of mechanical ventilation (dichotomized at above/below the upper quartile), length of total parental nutrition (dichotomized at above/below the upper quartile), PDA requiring medical treatment with indomethacin or ibuprofen (dichotomized yes/no), intrauterine growth restriction, necrotizing enterocolitis, and change in weight-for-height/length standard deviation score from birth to term-equivalent MRI.

\section{WM Injury}

Using T1- and T2-weighted images, subjects were categorized as having WM injury based on the presence of grade III/IV intraventricular hemorrhage and/or the presence of focal, extensive, or cystic WM lesions, as determined by a single neonatologist (41).

\section{Outcome Measures}

Preterm infants were assessed at $2 \mathrm{y}$ of age using the Bayley Scales of Infant and Toddler Development, $3^{\text {rd }}$ Edition (Bayley-III) (42) performed by a blinded psychometrician. Outcomes included Bayley-III cognitive, language, and motor composite scores. The Bayley-III also provides gross motor and fine motor subscale scores. Children were classified based on their composite scores into the following categories: normal $(\geq 95)$, mild $(<95$ and $\geq 80)$, moderate $(<80$ and $\geq 65)$, and severe impairment $(<65)(43)$. We utilized the ITSEA (23), a 166-item parent report measure that assesses the following four social-emotional domains: externalizing, internalizing, dysregulation, and competence. Only the ITSEA competence domain was used as it assesses attention and symptoms relevant to ASD, social competencies that have been described to be impaired among preterm infants (10). Higher scores indicate better performance and those scores that fall in the lower 10th percentile (most impaired) are categorized as "of concern."

\section{Data Analysis}

Data were analyzed using SPSS version 21 (IBM, New York, NY) and SAS 9.3 (SAS Institute, Cary, NC). Differences between excluded and included infants' baseline characteristics were assessed using $t$-tests for continuous variables and chi-square analyses for categorical variables. $\mathrm{FA}$ and $\mathrm{MD}$ values that were greater than three SD from the mean of the cohort were removed as outliers. FA and MD slopes were calculated for all ROIs and used as dependent variables in mixed random-effects models with embedded sequential Bonferroni adjustment for post hoc comparisons. Only infants with a term-equivalent scan and at least one additional scan collected at least $3 \mathrm{wk}$ prior were included. Mother and subject within mother were entered as random effects accounting for sibling correlation. Hemisphere, ROI, and hemisphere by ROI interaction effects were fixed factors. The following factors plus their interaction by ROI were included as covariates: GA, intrauterine growth restriction, length of ventilation in days, length of total parental nutrition in days, PDA requiring treatment, maternal use of antenatal steroids, treatment with postnatal steroids, change in standard deviation score, sepsis, and WM injury. As neither length of ventilation nor total parental nutrition were normally distributed, they were dichotomized, thresholding at the upper quartile. We repeated these same models with the ROIs combined into groups based on rates of myelination (early, intermediate, and late; detailed in the Supplementary Information online).

Diffusion measures at term-equivalent PMA were analyzed in relationship to developmental outcomes. All term-equivalent FA and MD measures were entered into a stepwise linear regression to determine independently significant variables. Significant variables $(P<0.05)$ were entered in linear mixed models adjusting for PMA at scan and sex. Clustering between twins/triplets was entered as a random effect. Adjustment for multiple comparisons was conducted by setting individual $\alpha$ levels for Bayley and ITSEA scales equal to 0.05 divided by the number of brain measures tested for that scale.

\section{SUPPLEMENTARY MATERIAL}

Supplementary material is linked to the online version of the paper at http:// www.nature.com/pr

\section{ACKNOWLEDGMENTS}

We express our appreciation to the following individuals who assisted with this project: Karen Lukas, Anthony Barton, Rachel Paul, Jessica Conners, Claudine Vavasseur, Han Tjoeng, Michael Wallendorf (who provided statistical consultation), Jim Alexopoulous, Jeanette Kenley, Joe Ackermann Jr., and Kayla Hannon. We also thank the families who participated.

\section{STATEMENT OF FINANCIAL SUPPORT}

This work was supported by the National Institutes of Health, Bethesda, Maryland (grant numbers R01 HD057098, UL1 TR000448, KL2 TR000450, and K02 NS089852); the McDonnell Center for Systems Neuroscience, St Louis, Missouri; the Intellectual and Developmental Disabilities Research Center at Washington University (NIH/NICHD Bethesda, Maryland, P30 HD062171); the Child Neurology Foundation, Minneapolis, Minnesota, and the Doris Duke Foundation, New York, New York.

\section{Disclosure: The authors have no financial disclosures to report.}

\section{REFERENCES}

1. Inder TE, Wells SJ, Mogridge NB, Spencer C, Volpe JJ. Defining the nature of the cerebral abnormalities in the premature infant: a qualitative magnetic resonance imaging study. J Pediatr 2003;143:171-9.

2. Hüppi PS, Maier SE, Peled S, et al. Microstructural development of human newborn cerebral white matter assessed in vivo by diffusion tensor magnetic resonance imaging. Pediatr Res 1998;44:584-90.

3. Partridge SC, Mukherjee P, Henry RG, et al. Diffusion tensor imaging: serial quantitation of white matter tract maturity in premature newborns. Neuroimage 2004;22:1302-14.

4. Kersbergen KJ, Leemans A, Groenendaal F, et al. Microstructural brain development between 30 and 40 weeks corrected age in a longitudinal cohort of extremely preterm infants. Neuroimage 2014;103:214-24.

5. Thompson DK, Inder TE, Faggian N, et al. Characterization of the corpus callosum in very preterm and full-term infants utilizing MRI. Neuroimage 2011;55:479-90.

6. Ball G, Counsell SJ, Anjari M, et al. An optimised tract-based spatial statistics protocol for neonates: applications to prematurity and chronic lung disease. Neuroimage 2010;53:94-102.

7. Thompson DK, Lee KJ, Egan GF, et al. Regional white matter microstructure in very preterm infants: predictors and 7 year outcomes. Cortex 2014;52:60-74.

8. Chau V, Brant R, Poskitt KJ, Tam EW, Synnes A, Miller SP. Postnatal infection is associated with widespread abnormalities of brain development in premature newborns. Pediatr Res 2012;71:274-9.

9. Chau V, Synnes A, Grunau RE, Poskitt KJ, Brant R, Miller SP. Abnormal brain maturation in preterm neonates associated with adverse developmental outcomes. Neurology 2013;81:2082-9.

10. Johnson S, Hollis C, Kochhar P, Hennessy E, Wolke D, Marlow N. Psychiatric disorders in extremely preterm children: longitudinal finding at age 11 years in the EPICure study. J Am Acad Child Adolesc Psychiatry 2010;49:453-63.e1.

11. Rogers CE, Anderson PJ, Thompson DK, et al. Regional cerebral development at term relates to school-age social-emotional development in very preterm children. J Am Acad Child Adolesc Psychiatry 2012;51: 181-91.

12. Wimberger DM, Roberts TP, Barkovich AJ, Prayer LM, Moseley ME, Kucharczyk J. Identification of "premyelination" by diffusion-weighted MRI. J Comput Assist Tomogr 1995;19:28-33.

13. Kinney HC, Brody BA, Kloman AS, Gilles FH. Sequence of central nervous system myelination in human infancy. II. Patterns of myelination in autopsied infants. J Neuropathol Exp Neurol 1988;47:217-34.

14. Cowan FM, de Vries LS. The internal capsule in neonatal imaging. Semin Fetal Neonatal Med 2005;10:461-74.

15. Kelly CE, Cheong JL, Molloy C, et al.; Victorian Infant Collaborative Study Group. Neural correlates of impaired vision in adolescents born extremely preterm and/or extremely low birthweight. PLoS One 2014;9:e93188. 
16. Thompson DK, Inder TE, Faggian N, et al. Corpus callosum alterations in very preterm infants: perinatal correlates and 2 year neurodevelopmental outcomes. Neuroimage 2012;59:3571-81.

17. Bonifacio SL, Glass HC, Chau V, et al. Extreme premature birth is not associated with impaired development of brain microstructure. J Pediatr 2010;157:726-32.e1.

18. Back SA, Riddle A, McClure MM. Maturation-dependent vulnerability of perinatal white matter in premature birth. Stroke 2007;38:Suppl 2:724-30.

19. Hoffmann O, Braun JS, Becker D, et al. TLR2 mediates neuroinflammation and neuronal damage. J Immunol 2007;178:6476-81.

20. Cheong JL, Thompson DK, Wang HX, et al. Abnormal white matter signal on MR imaging is related to abnormal tissue microstructure. AJNR Am J Neuroradiol 2009;30:623-8.

21. Lemmers PM, Toet MC, van Bel F. Impact of patent ductus arteriosus and subsequent therapy with indomethacin on cerebral oxygenation in preterm infants. Pediatrics 2008;121:142-7.

22. Miller SP, Mayer EE, Clyman RI, Glidden DV, Hamrick SE, Barkovich AJ. Prolonged indomethacin exposure is associated with decreased white matter injury detected with magnetic resonance imaging in premature newborns at 24 to 28 weeks' gestation at birth. Pediatrics 2006;117:1626-31.

23. Carter AS, Briggs-Gowan MJ. Infant Toddler Social and Emotional Assessment (ITSEA). San Antonio, TX: Psychological Corporation, Harcourt Assessment, 2006.

24. Travers BG, Adluru N, Ennis C, et al. Diffusion tensor imaging in autism spectrum disorder: a review. Autism Res 2012;5:289-313.

25. Noriuchi M, Kikuchi Y, Yoshiura T, et al. Altered white matter fractional anisotropy and social impairment in children with autism spectrum disorder. Brain Res 2010;1362:141-9.

26. Pellicano E. The development of executive function in autism. Autism Res Treat 2012;2012:146132.

27. Wolff JJ, Gu H, Gerig G, et al.; IBIS Network. Differences in white matter fiber tract development present from 6 to 24 months in infants with autism. Am J Psychiatry 2012;169:589-600.

28. Billeci L, Calderoni S, Tosetti M, Catani M, Muratori F. White matter connectivity in children with autism spectrum disorders: a tract-based spatial statistics study. BMC Neurol 2012;12:148.

29. Ben Bashat D, Kronfeld-Duenias V, Zachor DA, et al. Accelerated maturation of white matter in young children with autism: a high $b$ value DWI study. Neuroimage 2007;37:40-7.

30. Fischi-Gomez E, Vasung L, Meskaldji D-E, et al. Structural brain connectivity in school-age preterm infants provides evidence for impaired networks relevant for higher order cognitive skills and social cognition. Cereb Cortex 2015;25:2793-805.

31. Jahanshad N, Lee AD, Barysheva M, et al. Genetic influences on brain asymmetry: a DTI study of 374 twins and siblings. Neuroimage 2010;52: 455-69.

32. Dubois J, Hertz-Pannier L, Cachia A, Mangin JF, Le Bihan D, DehaeneLambertz G. Structural asymmetries in the infant language and sensorimotor networks. Cereb Cortex 2009;19:414-23.

33. Ortibus E, Verhoeven J, Sunaert S, Casteels I, de Cock P, Lagae L. Integrity of the inferior longitudinal fasciculus and impaired object recognition in children: a diffusion tensor imaging study. Dev Med Child Neurol 2012;54:38-43.

34. Skranes J, Vangberg TR, Kulseng S, et al. Clinical findings and white matter abnormalities seen on diffusion tensor imaging in adolescents with very low birth weight. Brain 2007;130(Pt 3):654-66.

35. Voineskos AN, Rajji TK, Lobaugh NJ, et al. Age-related decline in white matter tract integrity and cognitive performance: a DTI tractography and structural equation modeling study. Neurobiol Aging 2012;33:21-34.

36. Catani M, Jones DK, Donato R, Ffytche DH. Occipito-temporal connections in the human brain. Brain 2003;126(Pt 9):2093-107.

37. Ortibus E, Lagae L, Casteels I, Demaerel P, Stiers P. Assessment of cerebral visual impairment with the L94 visual perceptual battery: clinical value and correlation with MRI findings. Dev Med Child Neurol 2009;51:209-17.

38. Beaulieu C. The basis of anisotropic water diffusion in the nervous system - a technical review. NMR Biomed 2002;15:435-55.

39. Giménez M, Miranda MJ, Born AP, Nagy Z, Rostrup E, Jernigan TL. Accelerated cerebral white matter development in preterm infants: a voxelbased morphometry study with diffusion tensor MR imaging. Neuroimage 2008;41:728-34.

40. Kroenke CD, Bretthorst GL, Inder TE, Neil JJ. Modeling water diffusion anisotropy within fixed newborn primate brain using Bayesian probability theory. Magn Reson Med 2006;55:187-97.

41. Kidokoro H, Neil JJ, Inder TE. New MR imaging assessment tool to define brain abnormalities in very preterm infants at term. AJNR Am J Neuroradiol 2013;34:2208-14.

42. Bayley N. Bayley Scales of Infant and Toddler Development. 3rd edn. San Antonio, TX: Pearson Education, Inc., 2006.

43. Anderson PJ, De Luca CR, Hutchinson E, Roberts G, Doyle LW; Victorian Infant Collaborative Group. Underestimation of developmental delay by the new Bayley-III Scale. Arch Pediatr Adolesc Med 2010;164: 352-6. 